\title{
A CARGA DAS NEOPLASIAS NO BRASIL: MORTALIDADE E MORBIDADE HOSPITALAR ENTRE 2002-2004
}

\author{
Antonio Fernando Boing*, Silvia Angélica lópez Vargas, Alexandra Crispim Boing \\ Trabalho realizado no Departamento de Saúde Pública da Universidade Federal de Santa Catarina
}

\author{
*Correspondência \\ Universidade Federal de Santa \\ Catarina \\ Departamento de Saúde Pública \\ Campus Universitário \\ Florianópolis, SC, Brasil \\ Cep: $88040-900$
}

\begin{abstract}
RESUMO
OBjetivo. Descrever a morbidade hospitalar e a mortalidade por neoplasias no Brasil e regiões segundo gênero.

Métodos. Os dados de óbitos foram obtidos junto ao Sistema de Informações sobre Mortalidade e os de morbidade hospitalar no Sistema de Informações Hospitalares. Os óbitos foram categorizados segundo as localizações primárias do tumor, selecionadas de acordo com a décima revisão da Classificação Internacional de Doenças. Os dados populacionais são oriundos das estimativas intercensitárias do IBGE. O período de análise foi o triênio 2002-2004, o mais recente com dados de mortalidade no Brasil. Optou-se por calcular a média desse período para conferir maior estabilidade às taxas.

Resultados. Entre 2002 e 2004 ocorreram 405.415 óbitos por neoplasias no Brasil. As maiores taxas de mortalidade foram identificadas nas regiões Sul e Sudeste. Entre os homens, o câncer de traquéia, brônquios e pulmões foi a neoplasia maligna que apresentou maior mortalidade e entre as mulher foi o câncer de mama. Este agravo e o câncer de colo uterino foram os que mais demandaram internações, e a leucemia apresentou o maior custo médio e custo total em internações.
\end{abstract}

Conclusöes. A carga das neoplasias é extremamente elevada no Brasil e medidas públicas de caráter populacional devem ser priorizadas para o efetivo controle da morbidade e da mortalidade por este agravo.

UnItermos: Neoplasias. Mortalidade. Morbidade. Brasil.

\section{INTRODUÇÃo}

Durante o século $X X$, o Brasil e o mundo passaram por profundas alterações em seus perfis epidemiológico e demográfico. Concomitante ao declínio das taxas de mortalidade por doenças infecciosas houve aumento substancial na expectativa de vida da população e na proporção de mortes por doenças crônicas. Estas transformações têm ocorrido ao longo das últimas décadas com padrões e intensidades distintas entre as regiões geográficas e os estratos sociais dos países ${ }^{1-2}$.

A Organização Mundial de Saúde estimou que em 2005 ocorreram 35 milhões de mortes por doenças crônicas no mundo, sendo que aproximadamente 7,6 milhões, ou $21,7 \%$, corresponderam às neoplasias ${ }^{3}$. Caso medidas para o controle do câncer não sejam tomadas, 84 milhões de pessoas irão morrer nos próximos dez anos por esta causa. A tendência também é de acréscimo na incidência das neoplasias. Um aumento de 50\% num prazo de quinze anos é estimado, chegando-se a um total de 15 milhões de novos casos em 20204.

No Brasil, desde o ano 2000, as neoplasias correspondem à segunda causa de morte, atrás apenas das doenças do aparelho circulatório e superando o total de óbitos por causas externas 5 . Entre 1993 e 1999 houve uma variação percentual anual na mortalidade por câncer no Brasil de 2,68\% entre as mulheres e de 2,79\% entre os homens ${ }^{6}$. Estes valores são superiores aos observados nos períodos 1979-1985 e 1986-1992. Quanto à incidência, o Instituto Nacional do Câncer (INCA) estimou que em 2006 ocorram 234.570 novos casos de câncer no sexo masculino e 237.480 no sexo feminino no Brasil, sendo, à exceção de pele não melanoma, o câncer de mama feminina e o de próstata os mais incidentes ${ }^{7}$.

O presente estudo objetiva descrever a magnitude da mortalidade e da morbidade hospitalar por neoplasias no Brasil segundo cada localização primária do tumor. A análise da carga da doença é essencial para serem discutidas as estratégias de prevenção, diagnóstico precoce, tratamento e atenção paliativa das neoplasias.

\section{Métodos}

Para descrever o perfil de mortalidade e de morbidade hospitalar por neoplasias foram obtidos dados a partir do Sistema de Informações sobre Mortalidade (SIM) ${ }^{5}$ e do Sistema de Informações Hospitalares $(S \mid H)^{8}$, respectivamente. Os dados são de domínio público, acesso livre e disponibilizados pelo Ministério da Saúde. Os dados hospitalares são referentes às internações realizadas nas unidades participantes do SUS (públicas ou particulares conveniadas). Estima-se que elas correspondam a $70 \%$ do total de internações realizadas no Brasil|?.

As taxas de mortalidade foram calculadas para cada uma das localizações primárias do tumor codificando-se a causa básica de morte de acordo com a décima revisão da Classificação Internacional de Doenças (CID-10). Os dados foram estratificados segundo o gênero e as cinco grandes regiões do Brasil (Norte, Nordeste, Centro-Oeste, Sudeste e Sul). Os dados calculados referem-se à 


\begin{tabular}{|c|c|c|c|c|c|c|c|c|c|c|c|c|}
\hline \multirow[t]{2}{*}{ Neoplasias } & \multicolumn{2}{|c|}{ Norte } & \multicolumn{2}{|c|}{ Nordeste } & \multicolumn{2}{|c|}{ Sudeste } & \multicolumn{2}{|c|}{ Sul } & \multicolumn{2}{|c|}{ Centro-oeste } & \multicolumn{2}{|c|}{ Brasil } \\
\hline & Fem & Masc & Fem & Masc & Fem & Masc & Fem & Masc & Fem & Masc & Fem & Masc \\
\hline Lábio, cavidade oral e faringe & 0,48 & 1,61 & 0,97 & 2,71 & 1,26 & 6,44 & 1,24 & 6,88 & 1,09 & 4,30 & $|$,$| |$ & 4,93 \\
\hline Esôfago & 0,35 & 1,25 & 0,94 & 2,21 & 1,67 & 6,58 & 3,12 & 10,48 & 1,07 & 4,05 & 1,54 & 5,34 \\
\hline Estômago & 2,92 & 5,66 & 2,78 & 5,04 & 5,62 & 11,14 & 5,63 & 12,24 & 3,23 & 7,30 & 4,46 & 8,90 \\
\hline Cólon, reto e ânus & 1,45 & 1,22 & 2,09 & 1,68 & 7,73 & 6,93 & 7,96 & 7,57 & 3,91 & 3,59 & 5,45 & 4,87 \\
\hline Fígado e vias biliares intrahepáticas & 1,82 & 2,54 & 2,57 & 2,50 & 3,07 & 4,11 & 3,59 & 4,76 & 2,32 & 3,26 & 2,86 & 3,57 \\
\hline Pâncreas & 0,94 & 1,18 & 1,53 & $|, 4|$ & 3,64 & 3,70 & 4,38 & 4,69 & 2,06 & 2,54 & 2,84 & 2,93 \\
\hline Laringe & 0,16 & 1,05 & 0,22 & 1,35 & 0,42 & 3,87 & 0,54 & 4,26 & 0,43 & 2,05 & 0,36 & 2,87 \\
\hline Traquéia, brônquios e pulmões & 3,11 & 5,57 & 3,32 & 5,66 & 7,04 & 15,62 & 9,56 & 23,59 & 5,45 & 10,03 & 5,96 & 12,83 \\
\hline Pele & 0,06 & 0,15 & 0,16 & 0,22 & 0,66 & 0,81 & 1,20 & $\mid, 61$ & 0,36 & 0,45 & 0,53 & 0,68 \\
\hline Mama & 3,67 & 0,03 & 5,97 & 0,09 & $|3,9|$ & 0,11 & 13,64 & 0,08 & 7,67 & 0,04 & 10,44 & 0,09 \\
\hline Colo do útero & 5,68 & - & 4,35 & - & 4,45 & - & 5,47 & - & 5,04 & - & 4,71 & - \\
\hline Corpo e partes não especificadas do útero & $|, 6|$ & - & 2,30 & - & 3,56 & - & 3,74 & . & 2,88 & - & 3,04 & - \\
\hline Ovário & 0,89 & - & I,47 & . & 3,24 & - & 3,41 & - & 2,03 & - & 2,51 & - \\
\hline Próstata & - & 4,53 & - & 7,54 & - & 12,34 & - & 13,44 & - & 9,12 & - & $|0,3|$ \\
\hline Bexiga & 0,20 & 0,52 & 0,43 & 0,85 & 1,09 & 2,52 & 1,07 & 2,99 & 0,58 & 1,30 & 0,80 & 1,88 \\
\hline Meninge, encéfalo e outras partes do SNC & 1,17 & 1,45 & 2,04 & 2,16 & 3,61 & 4,11 & 4,15 & 5,05 & 2,98 & 3,31 & 3,02 & 3,44 \\
\hline Linfoma não-Hodgkin & 0,50 & 0,92 & 0,88 & 1,20 & 1,97 & 2,45 & 2,02 & 2,73 & 1,16 & 1,85 & $|, 5|$ & 1,98 \\
\hline Mieloma múltiplo e câncer de plasmócitos & 0,18 & 0,34 & 0,45 & 0,48 & 1,25 & 1,26 & 1,09 & 1,23 & 0,77 & 0,78 & 0,89 & 0,93 \\
\hline Leucemia & 1,61 & 2,10 & 1,79 & 2,24 & 2,92 & 3,49 & 3,11 & 4,10 & 2,44 & 3,16 & 2,50 & 3,10 \\
\hline In situ, benigna ou de Comportamento incerto & 1,29 & 1,38 & 0,89 & 0,87 & 1,07 & $|, 0|$ & 0,93 & 0,96 & 0,53 & 0,49 & 0,98 & 0,96 \\
\hline Outras neoplasias malignas & 7,64 & 8,34 & 10,59 & 10,03 & 15,90 & 16,67 & 16,87 & 18,65 & 12,39 & 13,02 & 13,69 & 14,19 \\
\hline Total & 35,74 & 39,83 & 45,75 & 48,25 & 84,09 & 103,17 & 92,74 & $|25,3|$ & 58,40 & 70,64 & 69,19 & 83,80 \\
\hline
\end{tabular}

SNC: Sistema Nervoso Central

média do triênio 2002-2004, o período mais recente com dados de mortalidade disponíveis em nível nacional. Optou-se por calcular a média desse período para conferir maior estabilidade às taxas, minimizando o impacto de possíveis variações aleatórias. Para o cálculo das taxas de mortalidade foram utilizadas as estimativas populacionais disponibilizadas pelo IBGE ${ }^{10}$ para os respectivos anos.

Quanto à morbidade hospitalar foram investigados o total de internações por neoplasias, a média de permanência do paciente no hospital, o valor médio por internação e o valor total anual do gasto decorrente de cada neoplasia. Os valores também são referentes à média do período 2002-2004.

\section{Resultados}

Entre 2002 e 2004 ocorreram 405.415 óbitos por neoplasias no Brasil. Cerca de 52,13\% desses óbitos aconteceram na região Sudeste e 20,96\% na região Sul. As regiões Nordeste (17,16\%), Centro-Oeste $(5,88 \%)$ e Norte $(3,86 \%)$ aparecem em seguida. Já a taxa de mortalidade por neoplasias foi maior na região Sul (108,83 por 100 mil habitantes) em relação às demais (93,43 por 100 mil hab. no Sudeste; 64,50 por 100 mil hab. no Centro-Oeste; 46,98 por 100 mil hab. no Nordeste e 37,8I por 100 mil hab. no Norte). Analisando-se o Brasil como um todo, a taxa de mortalidade foi de 76,39 por 100 mil hab.
A Tabela I descreve a mortalidade por neoplasias segundo 0 gênero, as localizações primárias do tumor e as regiões do país. Pode-se observar que o câncer de maior mortalidade entre as mulheres foi o de mama, com 10,44 mortes por 100 mil mulheres. A taxa de mortalidade por câncer de mama mais elevada foi encontrada na região Sudeste, existindo diferenças entre as taxas extremas (Sudeste e Norte) de 3,8 vezes. Ainda quanto ao gênero feminino observa-se que houve no período elevada taxa de mortalidade por câncer de traquéia, brônquios e pulmões, sendo maior na região Sul.

Entre os homens, o câncer de traquéia, brônquios e pulmões foi o que apresentou maior mortalidade, com taxa de 12,83 óbitos por 100 mil homens. As maiores taxas foram encontradas nas regiões Sul e Sudeste, havendo diferenças entre as taxas extremas na ordem de 4,2 vezes. A segunda maior taxa de mortalidade por neoplasias em homens brasileiros foi por câncer de próstata, com 10,31 mortes por 100 mil homens.

A Figura | apresenta as dez localizações topográficas com maiores taxas de mortalidade nas regiões brasileiras e no país agrupando-se os gêneros. Observa-se certa variação na ordem dos sítios com maior mortalidade entre as regiões, porém em todas o câncer de traquéia, brônquios e pulmão e de estômago são majoritários. 
Tabela 2-Média anual de internaçōes, média de permanência (dias), valor médio por internação ( $R$ \$) e média anual do valor total pago em internaçōes ( $\mathbf{R}$ ) segundo a localização primária do tumor. Brasil, 2002-2004

\begin{tabular}{|c|c|c|c|c|}
\hline Neoplasia & $\begin{array}{l}\text { Média anual de } \\
\text { internaçôes }\end{array}$ & $\begin{array}{c}\text { Média de } \\
\text { permanência(dias) }\end{array}$ & $\begin{array}{l}\text { Valor médio por } \\
\text { internaçáo(R\$) }\end{array}$ & $\begin{array}{l}\text { Média anual do valor tota } \\
\text { pago em internações (R\$) }\end{array}$ \\
\hline Lábio, cavidade oral e faringe. & 23.932 & 7,3 & 747,94 & $17.899 .803,99$ \\
\hline Esôfago & 11.549 & 9,7 & $1.011,81$ & | $1.685 .378,33$ \\
\hline Estômağo & 16.704 & 9,8 & $1.234,90$ & $20.628 .105,79$ \\
\hline Cólon, reto e ânus & 27.875 & 8,1 & $|| 57,.0 \mid$ & $32.252 .290,60$ \\
\hline Fígado e vias biliares intra-hepát & 3.163 & 8,9 & 643,29 & $2.034 .722,44$ \\
\hline Pâncreas & 2.750 & $|1|$, & $1.107,46$ & $3.045 .874,87$ \\
\hline Laringe & 6.889 & 6,8 & 880,63 & $6.066 .944,53$ \\
\hline Traquéia brônquios e pulmões & 11.813 & 10,6 & 871,96 & $|0.300 .70|, 43$ \\
\hline Pele & 12.877 & 4,5 & 658,29 & $8.476 .832,58$ \\
\hline Mama & 35.566 & 4,3 & 543,69 & $|9.337 .062,9|$ \\
\hline Colo do útero & 29.441 & 4,7 & 590,13 & $17.373 .889,45$ \\
\hline Corpo e partes não especificadas do útero & 27.276 & 4,1 & 572,92 & $15.626 .892,63$ \\
\hline Próstata & 12.135 & 6,7 & $1.077,87$ & $\mid 3.079 .916,80$ \\
\hline Bexiga & 5.776 & 7,3 & 893,03 & $5.157 .818,67$ \\
\hline $\begin{array}{l}\text { Neoplasia maligna das meninges, do encéfalo e de outras } \\
\text { partes do sistema nervoso central }\end{array}$ & 13.137 & 12,0 & $1.701,16$ & $22.348 .768,44$ \\
\hline Linfoma não-Hodgkin & 6.636 & 9,9 & $1.020,96$ & $6.775 .398,66$ \\
\hline Leucemia & 16.717 & 11,6 & $2.101,43$ & $35.128 .852,49$ \\
\hline $\begin{array}{l}\text { Neoplasia in-situ, benigna e de comportamento } \\
\text { incerto ou desconhecido }\end{array}$ & 168.364 & 3,9 & 478,04 & $80.483 .916,54$ \\
\hline Todas as neoplasias & 561.725 & 6,0 & 759,47 & $426.610 .594,79$ \\
\hline
\end{tabular}

A média anual do valor pago em internações por neoplasias entre 2002-2004 no Brasil foi de quase meio bilhão de reais (Tabela 2). Dentre as neoplasias malignas, as internações por leucemia foram as que apresentaram maior valor médio por internação e o maior gasto total. As neoplasias in situ, benignas e de comportamento incerto ou desconhecido apesar de terem um gasto médio de internações relativamente baixo apresentaram alto gasto total em razão do grande volume de internações hospitalares. Câncer de mama, de colo de útero e de cólon, reto e ânus foram as neoplasias malignas que mais internações ocasionaram. A neoplasia maligna das meninges, do encéfalo e de outras partes do sistema nervoso central foi a que exigiu maior tempo de internação ( 12 dias em média), seguida das leucemias $(||, 6)$, pâncreas $(||, \mid)$ e traquéia, brônquios e pulmões $(10,6)$.

\section{Discussão}

As altas taxas de incidência e de mortalidade por câncer no mundo no início do século XXI, somadas à expectativa de acréscimo nos seus valores nas próximas décadas apontam para a necessidade urgente de se intensificar pesquisas e ações para o controle desse agravo. Nos países desenvolvidos, um em cada cinco óbitos é decorrente de neoplasias, proporção que equivale a 10\% nos países em desenvolvimento. Estes países, entretanto, já concentram cerca de 55\% dos novos casos de câncer que surgem anualmente no mundo".
Durante o período 2002-2004, o Brasil apresentou uma taxa de mortalidade por câncer equivalente a 76,39 por 100 mil hab., sendo maior no gênero masculino (83,80 por 100 mil hab.) em relação ao gênero feminino $(69,19$ por 100 mil hab.). Diferenças na mortalidade por neoplasias associadas ao tabaco (como pulmão, boca, faringe, bexiga, laringe e esôfago), de 2 a 8 vezes maiores entre os homens, explicam parcialmente essa desigualdade. Pesquisas de base populacional indicam que o tabagismo é mais prevalente entre os homens, no entanto, é preocupante que 0 número de mulheres fumantes tem crescido no Brasil e a experimentação do cigarro tem acontecido em idades cada vez mais jovens ${ }^{12-13}$. O câncer de pulmão, altamente associado ao tabaco, foi entre 2002 e 2004 o primeiro em mortes por neoplasias entre os homens e o segundo entre as mulheres. Sendo o consumo de tabaco a maior causa prevenível de óbito em todo o mundo, a ação da sociedade e do governo para o seu controle deve ser intensa e duradoura $^{14}$. A recente aprovação no Congresso Nacional da Convenção Quadro para o Controle do Tabaco, após superação de grande resistência da indústria tabagista, representou um importante passo com vistas à redução no consumo de tabaco no Brasil. Porém, entidades da área da saúde e a própria sociedade devem permanecer vigilantes para garantir a execução das ações previstas no acordo e avançar no controle do tabaco. 
Figura I - Taxa de mortalidade segundo a localização primária do tumor no período 2002-2004. A) Região Norte; B) Região Nordeste; C) Região Sudeste; D) Região Sul;E) região Centro-Oeste; F) Brasil

(A)

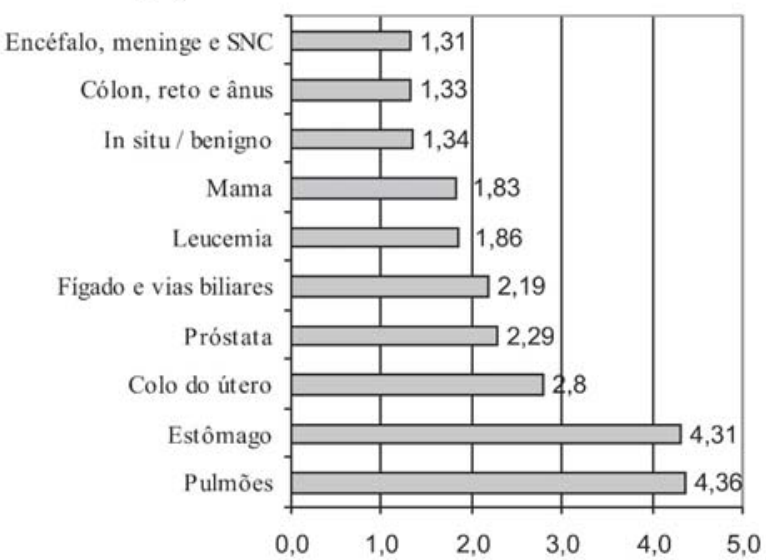

(C)

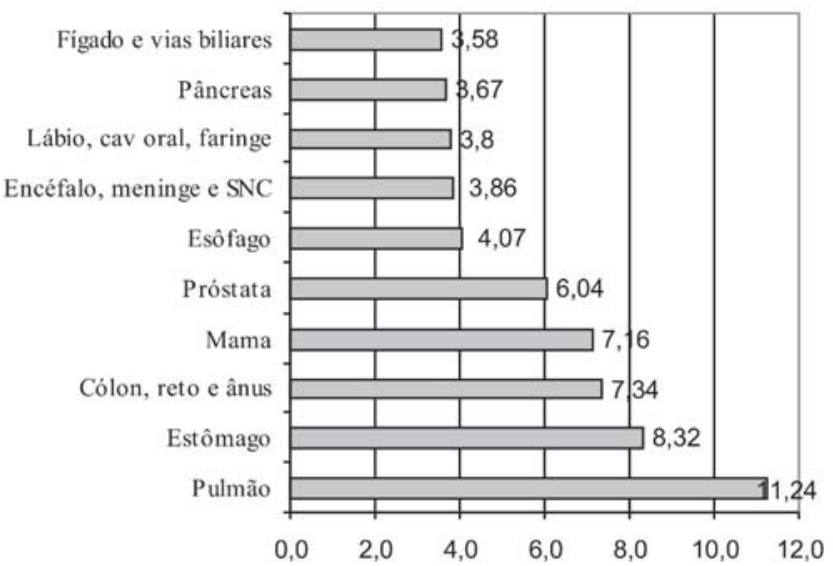

(E)

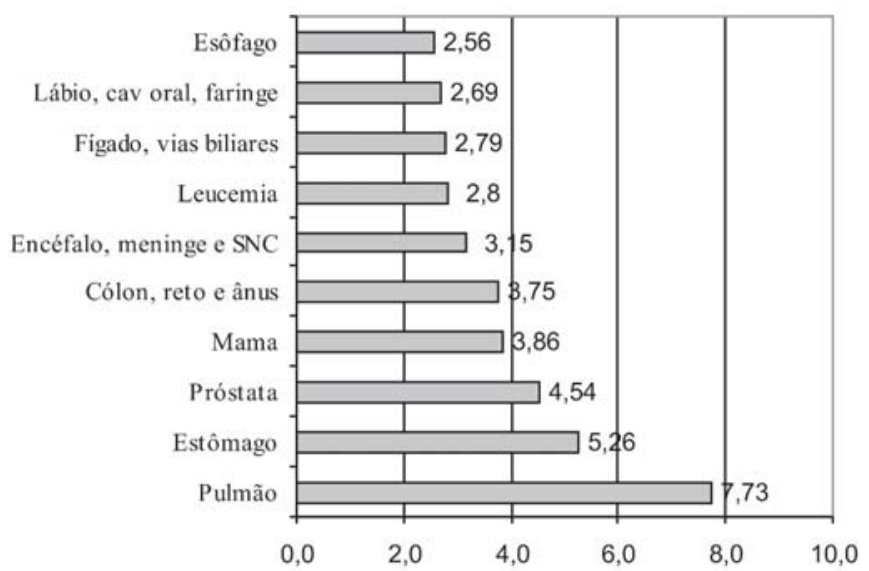

(B)

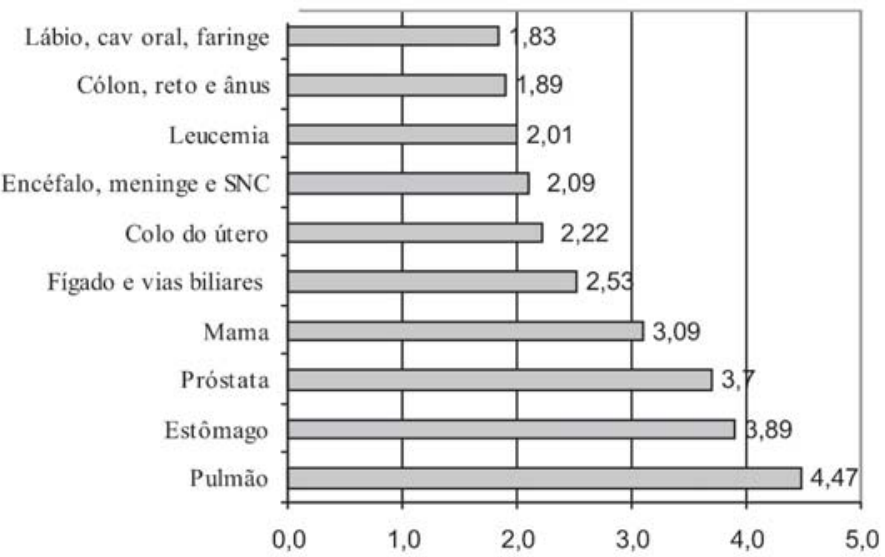

(D)

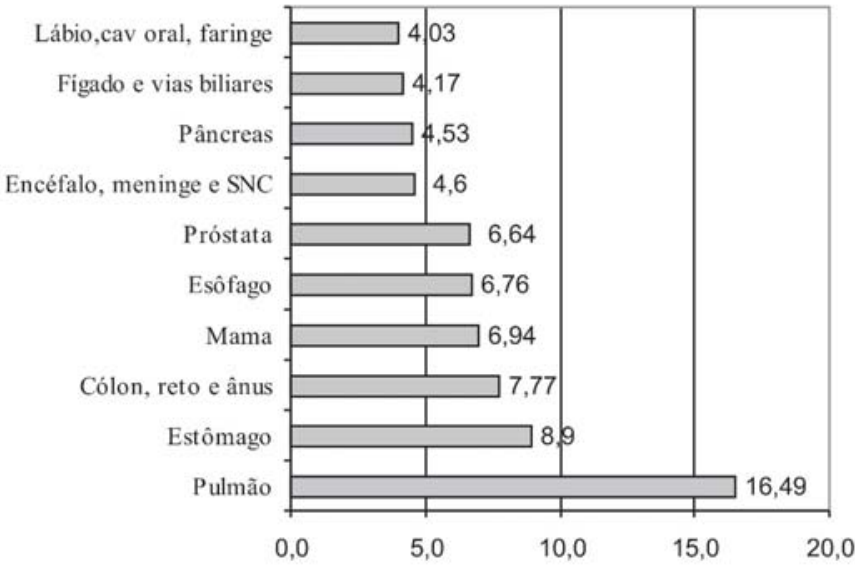

(F)

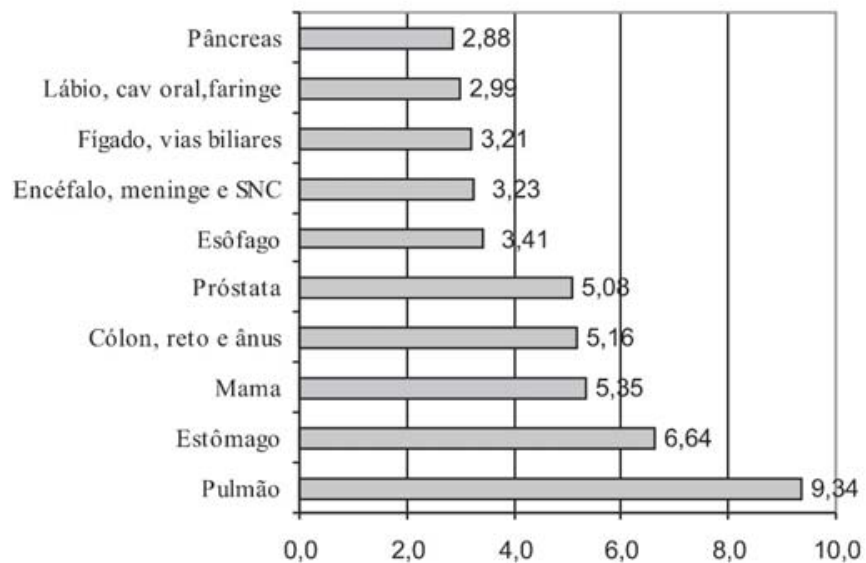


O câncer de mama foi o que apresentou a maior taxa de mortalidade entre as mulheres das regiões Sul, Sudeste, CentroOeste e Nordeste. Como houve no Brasil, nos últimos anos, um aumento não só na incidência, mas também na mortalidade por câncer de mama, os serviços de saúde devem aperfeiçoar o acesso ao diagnóstico precoce e à terapia apropriada ${ }^{15-16}$. Recentemente uma revisão sistemática que compreendeu dois ensaios clínicos randomizados com quase 400 mil mulheres apontou que 0 autoexame das mamas não foi capaz de reduzir a mortalidade por esta neoplasia, não sendo esta técnica recomendada para o seu rastreamento ${ }^{17}$. Não há ensaios clínicos randomizados investigando o exame clínico. Existe, portanto, necessidade de estruturação dos serviços de saúde com mamógrafos para atender à demanda e permitir um diagnóstico precoce da neoplasia.

Apesar de contar com sistema de prevenção relativamente simples e com custo reduzido, o câncer de colo de útero figurou como um dos tumores que mais mataram as mulheres no Brasil entre 2002-2004, tendo impacto singular na região Norte. Restrepo et al. ${ }^{18}$ relacionaram as diferenças de incidência por câncer de colo de útero encontradas entre regiỗes e dentro de países da América Latina e Caribe ao grau de desenvolvimento econômico e à oferta de serviços de saúde nas localidades. No Brasil, o acesso da população aos serviços de saúde para o diagnóstico do câncer de colo de útero apresenta grande variaçãa regional. Estados da região Norte, por exemplo, apresentaram baixa razão de exame citopatológico cervico-vaginal em mulheres de 25 a 59 anos em 2003-2004'9. Em diversos países desenvolvidos os programas de rastreamento populacional através da citologia vaginal têm tido impacto na redução da morbidade e da mortalidade por câncer de colo de útero ${ }^{20-21}$. O desafio para os países em desenvolvimento, inclusive o Brasil, é aumentar a cobertura dos programas de prevenção do câncer de colo de útero. O diagnóstico de lesões precursoras do tumor e o tratamento de lesões mais avançadas é fundamental para que se evite a progressão da doença para formas mais invasoras e para que se obtenha um melhor prognóstico. É importante observar, entretanto, como aponta Wünsch-Filho (2002)22 que o acesso a esses serviços de consulta não se dá pela simples escolha do indivíduo, mas está estruturalmente determinado.

Em relação à mortalidade por câncer de estômago, houve no Brasil ao longo dos últimos anos cessação na tendência de acréscimo ${ }^{15,22}$. O INCA (Instituto Nacional do Câncer) ${ }^{15}$ apontou uma queda da mortalidade por esse tumor estatisticamente significante entre as mulheres no início da década de 1980 e estabilidade para ambos os gêneros até 1999. Analisando-se homens e mulheres em conjunto identifica-se que o câncer de estômago foi o segundo com maior taxa de mortalidade em todas as regiões do país. Fatores como a adequada conservação dos alimentos em geladeiras e congeladores, além do consumo de frutas, vegetais, vitaminas $\mathrm{A}, \mathrm{C}$ e E associados à restrição de comida defumada e conservada em vinagre e sal são apontados como elementos protetores para o câncer gástrico e devem ser promovidos para o controle do tumor ${ }^{23}$.

Desde 1979, primeiro ano de cobertura nacional do SIM, a mortalidade por câncer de próstata tem variado positivamente no Brasill ${ }^{15}$. Também a incidência da morbidade vem crescendo, possivelmente inclusive por melhorias nos sistemas de registro, avanço dos métodos de diagnóstico e envelhecimento da população ${ }^{24}$.
No presente estudo identificou-se que apenas o câncer de traquéia, de brônquios e de pulmões matou mais homens que o câncer de próstata. O conhecimento sobre as causas desta neoplasia ainda é limitado, porém já se sabe que uma alimentação rica em frutas, verduras e fibras e limitada em gordura contribui na sua prevenção ${ }^{25}$. O desafio para um melhor controle da doença passa pelo desenvolvimento de melhores métodos de rastreamento, na apropriada determinação dos indivíduos que precisam realizar biópsia e numa melhor predição da evolução dos casos de acordo com as terapias instituídas ${ }^{26}$.

Não foi objetivo desse estudo comparar as diferenças de mortalidade entre as regiões, mas sim descrever a carga de doença nessas localidades para se conhecer a magnitude real das taxas de mortalidade de cada neoplasia nas regiões. Portanto, optou-se pela não-padronização dos coeficientes. Além da estrutura etária, podem estar modulando as variações regionais na mortalidade por neoplasias no Brasil as diferenças socioeconômicas, os diferentes padrões de dieta, de prevalência de tabagismo e etilismo, exposições ambientais e ocupacionais. Variações relacionadas aos serviços de saúde, como acesso ao diagnóstico e tratamento, qualidade no atendimento, acessibilidade, eqüidade, universalidade e cobertura também devem ser consideradas. Por fim, há acentuada variação na qualidade dos registros de mortalidade no Brasil. As regiões Sul e Sudeste apresentam menor proporção de óbitos classificados como mal definidos em relação ao Centro-Oeste, Nordeste e $\mathrm{Norte}^{27}$. Dessa maneira, a magnitude da mortalidade por todos os agravos, inclusive por neoplasias, são subestimados nestas regiões.

As internações hospitalares no SUS por neoplasias superaram 500 mil por ano, com um gasto, também anual, de quase meio bilhão de reais. Deve-se ressaltar que esses elevados valores correspondem apenas às internações no SUS e não consideram os gastos ambulatoriais e os custos indiretos, que seriam aqueles relacionados à perda de produtividade dos pacientes. A leucemia foi a neoplasia maligna que apresentou o maior custo médio, maior custo total e foi a segunda em média de permanência hospitalar. Apesar de não ser o que mais mata, esse agravo exige uma terapêutica de grande investimento com combinações de drogas, transfusões e internações. $O$ câncer de mama e de colo uterino foram os que mais demandaram internações, apesar de serem doenças em que a prevenção e o diagnóstico precoce estão dentro das possibilidades dos serviços de saúde. Métodos de rastreamento dessas neoplasias têm se mostrado muito mais racionais do ponto de vista econômico, além de evitar mortes, sofrimentos e problemas psicológicos para 0 paciente e a família ${ }^{28-29}$.

Segundo Danaei et al. ${ }^{30}$, do total de sete milhões de óbitos por câncer ocorridos no ano de 2001 , cerca de $35 \%$, ou 2,3 milhões de mortes, podem ser atribuídas a nove fatores de risco passíveis de controle. Dentre os principais estão o tabagismo, o etilismo, o baixo consumo de frutas e verduras e a obesidade. Rose ${ }^{31}$ ressaltou que a quantidade de pessoas excessivamente expostas a fatores de risco está associada à exposição média de toda a população a esses fatores. Ou seja, mais do que simples opções individuais, a prática regular de exercícios físicos, o tabagismo, o etilismo, a dieta balanceada, etc. são fortemente influenciadas por questões econômicas, sociais e culturais. Portanto, a redução da mortalidade e da 
BOING AF ET AL.

morbidade por neoplasias no Brasil deve estar pautada por políticas públicas de abrangência populacional, criando a possibilidade das pessoas optarem pelos hábitos de vida mais saudáveis.

\section{Conclusão}

As neoplasias apresentam altas taxas de mortalidade no Brasil e elevados custos clínicos assistenciais. A magnitude dos agravos varia de acordo com a localização primária do tumor, o gênero do paciente e a região do país.

Diversos fatores de risco e de prevenção para as neoplasias, e também de métodos terapêuticos, já estão estabelecidos e tais conhecimentos devem ser aplicados de maneira universal e equânime com vistas ao controle deste agravo.

\section{Conflito de interesse: não há.}

\section{SUMMARY}

\section{THE BURDEN OF NEOPLASM IN BRAZIL: MORTALITY AND HOSPITAL MORBIDITY FROM 2002 TO 2004}

OBJECTNE. To describe mortality and the hospital morbidity by neoplasias in Brazil and regions according to gender.

METHODS. Data of deaths were obtained from the Mortality Information System and of hospital morbidity from the Hospital Information System. Deaths were categorized according to primary tumor sites, selected in accordance with the tenth revision of the International Classification of Diseases. The population data were drawn from the inter census estimates of the IBGE (Brazilian Institute of Geography and Statistics). The period of analysis was the triennial 2002-2004, with the most recent mortality data in Brazil. The average of this period was calculated to ensure greater stability of the rates.

RESULTS. Between 2002 and 2004, 405,415 deaths from neoplasias occurred in Brazil. The highest rates of mortality were identified in the South and South-East regions. For men, cancer of the trachea, bronchi and lungs were the malignant neoplasias with the highest mortality rate while for women breast cancer was highest. Breast cancer and cancer of the uterine cervix are those requiring the largest number of in-hospital admissions. In internments, leukemia presented the highest average cost and total cost.

CONCLUSION. The burden of neoplasms is extremely high in Brazil and public policies focused on the population must be given priority for an effective control of mortality and morbidity. [Rev Assoc Med Bras 2007; 53(3): 317-22]

KEY wORDS: Neoplasm. Mortality. Morbidity. Brazil.

\section{REFERÊNCIAS}

I. Barreto ML, Carmo EH. Mudanças em padrões de morbimortalidade: conceitos e métodos. In: Monteiro CA, organiadores. Velhos e novos males de saúde no Brasil: a evolução do país e de suas doenças. São Paulo: Hucitec; 1995

2. OPAS (Organização Pan-americana de Saúde). Indicadores Básicos para a Saúde no Brasil: conceitos e aplicações. Brasília: OPAS; 2002.

3. WHO (World Health Organization). Preventing chronic diseases: a vital investment. Geneve: WHO; 2005

4. WHO (World Health Organization). Global cancer rates could increase by $50 \%$ to 15 million by 2020 . Disponível em: http://www.who.int/ mediacentre/releases/2003/ pr27/en. Acesso em: 0I out. 2006.
5. Brasil. Ministério da Saúde. Sistema de informações sobre mortalidade. Disponível em: http://tabnet.datasus.gov.br/cgi/sim/obtmap.htm. Acesso em: 20 set. 2006.

6. Brasil. Ministério da Saúde. Instituto Nacional de Câncer. Atlas de mortalidade por câncer no Brasil 1979-1999. Rio de Janeiro: INCA; 2002.

7. Brasil. Ministério da Saúde. Instituto Nacional de Câncer. Estimativa 2006: incidência de câncer no Brasil. Rio de Janeiro: INCA; 2005.

8. Brasil. Ministério da Saúde. Sistema de informações hospitalares. Disponível em: http://tabnet.datasus.gov.br/cgi/sih/rxmap.htm. Acesso em: 21 set. 2006.

9. OPAS (Organização Pan-americana de Saúde). Indicadores básicos para a saúde no Brasil: conceitos e aplicações. Brasília: OPAS; 2002.

10. Brasil. Instituto Brasileiro de Geografia e Estatística. Disponível em: http://www.ibge.gov.br. Acesso em: 20 set. 2006.

I I. WHO (World Health Organization). National cancer control programmes: policies and managerial guidelines. Roma: WHO; 2002.

12. Brasil. Instituto Nacional do Câncer. Por um mundo sem tabaco: mobilização da sociedade civil. Rio de Janeiro: INCA; 2004.

13. Szwarcwald CL, Viacava F, Vasconcellos MTL, Leal MC, Azevedo MO, Queiroz RSB, et al.. O Brasil em números. Radis. 2004; 23:14-33.

14. WHO (World Health Organization). Global action against cancer. Geneve: WHO; 2003.

15. Brasil. Ministério da Saúde. Instituto Nacional de Câncer. Atlas de mortalidade por câncer no Brasil 1979-1999. Rio de Janeiro: INCA; 2002.

16. Brasil. Ministério da Saúde. Câncer de mama: documento de consenso. Rio de Janeiro: INCA; 2004.

17. Kösters JP, Gøtzsche PC. Regular self-examination or clinical examination for early detection of breast cancer (Cochrane Review). The Cochrane Library.2006; I

18. Restrepo HE, Gonzalez J, Roberts E, Litvak J. Epidemiologia y control del cancer del cuello uterino en America Latina y el Caribe. Bol Of Sanit Panam 1987; 102:578-92.

19. Brasil. Ministério da Saúde. Pacto de atenção básica 2005. Disponível em: http://tabnet.datasus.gov.br/cgi/siab/pacto2005/pacmap. htm.Acesso em: 12 set. 2006.

20. Hakama M, Chamberlain J, Day NE, Miller AB, Prorok PC. Evaluation of screening programmes for gynaecological cancer. $\mathrm{Br} J$ Cancer. 1985;52:669-73.

21. Miller AB. Report on a workshop UICC project on evaluation of screening for cancer. Int J Cancer. 1990;46:76I-9.

22. Wünsh-Filho V, Moncau JE. Mortalidade por câncer no Brasil 19801995: padrõesregionais e tendências temporais. Rev Assoc Med Bras 2002:48:250-7.

23. Abreu E. A prevenção primária e a detecção do câncer de estômago. Cad Saúde Pública. 1997; |3(supl | ): |05-8.

24. INCA (Instituto Nacional do Câncer). Câncer de próstata. Disponível em: http://www.inca.gov.br/conteudo view.asp?ID=339. Acesso em: 12 set 2006.

25. Grönberg H. Prostate cancer epidemiology. Lancet. 2003; 361:859-64.

26. Brawer MK, Crawford ED, Fowler J, Lucia MS, Schroeder FH. Prostate cancer: epidemiology and screening. Rev Urol. 2000;2(Suppl 4):5-9.

27. Mello Jorge MHP, Gotlieb SLD. As condições de saúde no Brasil. Rio de Janeiro: Fiocruz; 2000.

28. Kemp C. Elias S, Gebrim LH, Nazário ACP, Baracat EC, Lima GR. Estimativa de custo do rastreamento mamográfico em mulheres no climatério. Rev Brass Ginecol Obstet. 2005;27:415-20.

29. Caetano R, Caetano CMM. Custo-efetividade no rastreamento do câncer cérvico-uterino no Brasil: um estudo exploratório. Rio de Janeiro: INCA; 2005.

30. Danaei G, Vander Hoorn S, Lopez AD, Murray CJ, Ezzati M. Causes of cancer in the world: comparative risk assessment of nine behavioural and environmental risk factors. Lancet. 2005;366(9499): 1784-93.

31. Rose G. The Strategy of Preventive Medicine. New York: Oxford University Press; 1992. 